\title{
An Extension of Darbo's Theorem and Its Application
}

\author{
A. Samadi and M. B. Ghaemi \\ Department of Mathematics, College of Basic Science, Karaj Branch, Islamic Azad University, Alborz, Iran
}

Correspondence should be addressed to M. B. Ghaemi; mghaemi@iust.ac.ir

Received 8 February 2014; Accepted 4 April 2014; Published 11 May 2014

Academic Editor: Chuanzhi Bai

Copyright (C) 2014 A. Samadi and M. B. Ghaemi. This is an open access article distributed under the Creative Commons Attribution License, which permits unrestricted use, distribution, and reproduction in any medium, provided the original work is properly cited.

Here, some extensions of Darbo fixed point theorem associated with measures of noncompactness are proved. Then, as an application, our attention is focused on the existence of solutions of the integral equation $x(t)=F\left(t, f\left(t, x\left(\alpha_{1}(t)\right)\right.\right.$, $\left.\left.x\left(\alpha_{2}(t)\right)\right),((T x)(t) / \Gamma(\alpha)) \times \int_{0}^{t}\left(u\left(t, s, \max _{[0, r(s)]}\left|x\left(\gamma_{1}(\tau)\right)\right|, \max _{[0, r(s)]}\left|x\left(\gamma_{2}(\tau)\right)\right|\right) /(t-s)^{1-\alpha}\right) d s, \quad \int_{0}^{\infty} v(t, s, x(t)) d s\right), \quad 0<\alpha \leq 1, t \in$ $[0,1]$ in the space of real functions defined and continuous on the interval $[0,1]$.

\section{Introduction}

The concept of measure of noncompactness plays very important role in describing differential and integral equations. It was introduced by Kuratowski [1] as follows:

$$
\begin{aligned}
& \alpha(S)=\inf \{\delta>0: \\
& \left.\qquad=\bigcup_{i=1}^{n} S_{i}, \operatorname{diam}\left(S_{i}\right) \leq \delta, 1 \leq i \leq n<\infty\right\},
\end{aligned}
$$

for bounded subsets $S$ of a metric space $X$. Darbo [2] used Kuratowski measure of noncompactness to generalize Shauder fixed point theorem to $k$-set contractive operators, which satisfy the condition $\alpha(T(A)) \leq k \alpha(A)$ for some $k \epsilon$ $[0,1)$. Up to now, other measures of noncompactness have been defined. In recent years many papers have been devoted to the problem of existence of solutions of integral equations, using the technique of measures of noncompactness and Darbo fixed point theorem (cf. [3-6]). Recently, the technique of measure of noncompactness has been used to obtain some extensions of Darbo fixed point theorem and the obtained results have important applications $[3,4]$. As some applications of the technique of measures of noncompactness and
Darbo fixed point theorem, the following integral equations have been considered in $[5,6]$, respectively:

$$
\begin{gathered}
x(t)=f(t)+\frac{(T x)(t)}{\Gamma(\alpha)} \int_{0}^{t} \frac{u\left(t, s, \max _{[0, r(s)]}|x(\tau)|\right)}{(t-s)^{1-\alpha}} d s, \\
0<\alpha \leq 1, \quad t \in[0,1], \\
x(t)=F(t, f(t, x(\alpha(t))), \\
\int_{0}^{\beta(t)} u(t, s, x(\gamma(s))) d_{s}(t, s), \\
\left.\int_{0}^{\infty} v(t, s, x(\delta(s))) d s\right), \quad t \in \mathbb{R}_{+} .
\end{gathered}
$$

Huang and Cao [7] have given a result to find the solution of the integral equation

$$
\begin{aligned}
x(t)= & q(t)+f_{1}\left(t, x\left(\alpha_{1}(t)\right), x\left(\alpha_{2}(t)\right)\right) \\
& +\frac{f_{2}\left(t, x\left(\beta_{1}(t)\right), x\left(\beta_{2}(t)\right)\right)}{\Gamma(\alpha)} \\
& \times \int_{0}^{t}(t-s)^{\alpha-1} f_{3}\left(t, s, x\left(\gamma_{1}(s)\right), x\left(\gamma_{2}(s)\right)\right) d s .
\end{aligned}
$$


In this paper, motivated and inspired by the integral equations (2), (3), and (4), we are going to prove a theorem on the existence of solutions of the integral equation

$$
\begin{gathered}
x(t)=F\left(t, f\left(t, x\left(\alpha_{1}(t)\right), x\left(\alpha_{2}(t)\right)\right),\right. \\
\frac{(T x)(t)}{\Gamma(\alpha)} \times \int_{0}^{t} u\left(t, s, \max _{[0, r(s)]}\left|x\left(\gamma_{1}(\tau)\right)\right|\right. \\
\left.\quad \max _{[0, r(s)]}\left|x\left(\gamma_{2}(\tau)\right)\right|\right) \\
\left.\quad \int_{0}^{\infty} v(t, s, x(t)) d s\right), \\
0<\alpha \leq 1, \quad t \in[0,1]
\end{gathered}
$$

in the Banach space $C(I)=C[0,1]$. Note that (5) has a rather general form and extends the integral equation (2). Our aim will be realized with help of the technique of measure of noncompactness. In Section 2, we present some definitions and preliminary results about the concept of measure of noncompactness. In Section 3, using the existent contractive condition in [8, Theorem 3.1] and the notion of shifting distance functions of [9], some generalizations of Darbo fixed point theorem are proved. In the last section, a result is proved concerning the existence of solutions for the integral equation (5).

\section{Preliminaries}

In this section, some definitions, notions, and results are presented which will be used in the next sections.

Assume that $E$ is a real Banach space with zero element $\theta$. The closed ball centered at $x$ with radius $r$ and the ball $B(\theta, r)$ are denoted by $B(x, r)$ and $B_{r}$, respectively. If $X$ is a nonempty subset of $E$, then we denote by $\bar{X}$ and $\operatorname{conv}(X)$ the closure and closed convex hull of $X$, respectively. Moreover, let $M_{E}$ indicate the family of all nonempty bounded subsets of $E$ and $N_{E}$ its subfamily consisting of all relatively compact subsets of $E$.

In our considerations, we use the following definition of the concept of measure of noncompactness.

Definition 1 (see [10]). A mapping $\mu: M_{E} \rightarrow[0, \infty)$ is called a measure of noncompactness if it satisfies the following conditions.

(1) The family $\operatorname{Ker} \mu=\left\{X \in M_{E}: \mu(X)=0\right\}$ is nonempty and $\operatorname{Ker} \mu \subseteq N_{E}$.

(2) $X \subseteq Y \Rightarrow \mu(X) \leq \mu(Y)$.

(3) $\mu(\bar{X})=\mu(X)$.

(4) $\mu(\operatorname{Conv}(X))=\mu(X)$.

(5) $\mu(\lambda X+(1-\lambda) Y) \leq \lambda \mu(X)+(1-\lambda) \mu(Y)$ for $\lambda \in[0,1]$.
(6) If $\left\{X_{n}\right\}$ is a sequence of closed sets from $M_{E}$ such that $X_{n+1} \subseteq X_{n}$ for $n=1,2, \ldots$ and $\lim _{n \rightarrow \infty} \mu\left(X_{n}\right)=0$, then $X_{\infty}=\bigcap_{n=1}^{\infty} X_{n}$ is nonempty.

The family Ker $\mu$ described in (1) is said to be the kernel of the measure of noncompactness $\mu$ and since $\mu\left(X_{\infty}\right)=$ $\mu\left(\bigcap_{n=1}^{\infty} X_{n}\right) \leq \mu\left(X_{n}\right)$, we infer that $\mu\left(\bigcap_{n=1}^{\infty} X_{n}\right)=0$. So, $X_{\infty} \in \operatorname{Ker} \mu$.

Let $C(I)=C([0,1])$ denote the Banach space of all real functions defined and continuous on the interval $[0,1]$ equipped with the norm

$$
\|x\|=\max \{|x(t)| ; t \in[0,1]\} .
$$

Fix a nonempty subset $X$ of $C([0,1])$. For $\varepsilon>0$ and $x \in X$ define

$$
\begin{gathered}
\omega(x, \varepsilon)=\sup \left\{\left|x\left(t_{1}\right)-x\left(t_{2}\right)\right| ;\right. \\
\left.t_{1}, t_{2} \in[0,1],\left|t_{2}-t_{1}\right| \leq \varepsilon\right\}, \\
\omega_{0}(X, \varepsilon)=\sup \{\omega(x, \varepsilon) ; x \in X\}, \\
\omega_{0}(X)=\lim _{\varepsilon \rightarrow 0} \omega(X, \varepsilon) .
\end{gathered}
$$

Banas and Lecko [11] showed that the function $\omega_{0}(X)$ is a measure of noncompactness in $C(I)$. Now, we state the following two important theorems which play a key role in the fixed point theory.

Theorem 2 (Schauder [12]). Let $U$ be a nonempty, bounded, closed, and convex subset of a Banach space E. Then, every continuous and compact map $F: U \rightarrow U$ has at least one fixed point in $U$.

Theorem 3 (Darbo [10]). Let $Q$ be a nonempty, closed, bounded, and convex subset of a Banach space $E$ and let $F$ : $Q \rightarrow Q$ be a continuous mapping. Assume that there exists a constant $k \in[0,1)$ such that $\mu(F X) \leq k \mu(X)$ for any nonempty subset $X$ of $Q$. Then, $F$ has a fixed point in $Q$.

The following definition of the concept of shifting distance functions will be used to generalize Darbo fixed point theorem.

Definition 4 (see [9]). Let $\psi, \phi:[0, \infty) \rightarrow \mathbb{R}$ be two functions. The pair of functions $(\phi, \psi)$ is said to be a pair of shifting distance functions, if the following conditions hold:

(i) for $u, v \in[0, \infty)$ if $\psi(u) \leq \phi(v)$, then $u \leq v$;

(ii) for $\left\{u_{n}\right\},\left\{v_{n}\right\} \subset[0, \infty)$ with $\lim _{n \rightarrow \infty} u_{n}=$ $\lim _{n \rightarrow \infty} v_{n}=w$, if $\psi\left(u_{n}\right) \leq \phi\left(v_{n}\right)$ for all $n$, then $w=0$.

Example 5 (see [9]). The conditions (i) and (ii) of the above definition are fulfilled for the functions $\psi, \phi:[0, \infty) \rightarrow \mathbb{R}$ defined by $\psi(t)=\ln ((1+2 t) / 2)$ and $\phi(t)=\ln ((1+t) / 2)$.

\section{Some Generalizations of Darbo Fixed Point Theorem}

In this section, we prove some generalizations of Darbo fixed point theorem. 
Now, using the notion of shifting distance functions of [9], we obtain our generalization of Darbo fixed point theorem as follows.

Theorem 6. Let $U$ be a nonempty, bounded, closed, and convex subset of the Banach space E. Moreover, assume that $T: U \rightarrow U$ is a continuous function such that

$$
\psi(\mu(T X)) \leq \phi(\mu(X))
$$

for any nonempty subset $X$ of $U$, where $\mu$ is an arbitrary measure of noncompactness and $\psi, \phi:[0, \infty) \rightarrow R$ are the pair of shifting distance functions. Then, $T$ has a fixed point in $U$.

Proof. Define the sequence $\left\{U_{n}\right\}$ by $U_{0}=U$ and $U_{n}=$ $\operatorname{Conv}\left(T U_{n-1}\right)$ for all $n \geq 1$. If there exists an integer $n_{0} \geq 0$ such that $\mu\left(U_{n_{0}}\right)=0$, then $U_{n_{0}}$ is relatively compact and since $T U_{n_{0}} \subseteq U_{n_{0}}$, thus Theorem 2 implies that $T$ has a fixed point. So suppose that $\mu\left(U_{n}\right)>0$ for all $n \geq 0$. Using (8) we get

$$
\begin{aligned}
\psi\left(\mu\left(U_{n+1}\right)\right) & =\psi\left(\mu\left(\operatorname{Conv}\left(T U_{n}\right)\right)\right) \\
& =\psi\left(\mu\left(T U_{n}\right)\right) \leq \phi\left(\mu\left(U_{n}\right)\right) .
\end{aligned}
$$

Due to condition (i) of Definition 4 and (9) we infer that $\left\{\mu\left(U_{n}\right)\right\}$ is a decreasing sequence of positive real numbers. Thus, there exists $r \geq 0$ such that $\mu\left(U_{n}\right) \rightarrow r$ as $n \rightarrow \infty$. So, in view of (9) and condition (ii) of Definition 4, we get $r=0$ and hence $\lim _{n \rightarrow \infty} \mu\left(U_{n}\right)=0$. Now, since $U_{n+1} \subseteq U_{n}$, $T U_{n} \subseteq U_{n}$, and $\mu\left(U_{n}\right) \rightarrow 0$ as $n \rightarrow \infty$, condition (6) of Definition 1 implies that $U_{\infty}=\bigcap_{n=1}^{\infty} U_{n}$ is nonempty, closed, convex, and invariant under the operator $T$ and belongs to Ker $\mu$. So, Theorem 2 completes the proof.

Taking $\psi=I$ in Theorem 6 , we have the following result.

Corollary 7. Let $U$ be a nonempty, bounded, closed, and convex subset of the Banach space E. Moreover, assume that $T: U \rightarrow U$ is a continuous function such that

$$
\mu(T X) \leq \phi(\mu(X))
$$

for any nonempty subset $X$ of $U$, where $\mu$ is an arbitrary measure of noncompactness and $\phi:[0, \infty) \rightarrow \mathbb{R}$ is a function such that

(a) for $\left\{u_{n}\right\},\left\{v_{n}\right\} \subset[0, \infty)$ with $\lim _{n \rightarrow \infty} u_{n}=$ $\lim _{n \rightarrow \infty} v_{n}=w$, if $u_{n} \leq \phi\left(v_{n}\right)$ for all $n$, then $w=0$;

(b) for $u, v \in[0, \infty)$ if $u \leq \phi(v)$, then $u \leq v$.

Then, $T$ has a fixed point in $U$.

Using Proposition 9 proved in [9] and Theorem 6, we have the following result.

Corollary 8. Let $U$ be a nonempty, bounded, closed, and convex subset of the Banach space E. Moreover, assume that $T: U \rightarrow U$ is a continuous function such that

$$
\psi(\mu(T X)) \leq \psi(\mu(X))-\varphi(\mu(X))
$$

for any nonempty subset $X$ of $U$, where $\mu$ is an arbitrary measure of noncompactness and $\phi, \psi:[0, \infty) \rightarrow[0, \infty)$ are two nondecreasing and continuous functions satisfying $\psi(t)=$ $\varphi(t)=0$ if and only if $t=0$. Then, Thas a fixed point in $U$.

Now, motivated and inspired by the contractive condition in [8, Theorem 3.1], we present another generalization of Darbo fixed point theorem as follows.

Theorem 9. Let $U$ be a nonempty, bounded, closed, and convex subset of the Banach space E. Moreover, assume that $T: U \rightarrow$ $U$ is a continuous function such that

$$
\psi(\mu(T X)) \leq \varphi(\mu(X))-\theta(\mu(X))
$$

for any nonempty subset $X$ of $U$, where $\mu$ is an arbitrary measure of noncompactness and $\psi, \phi, \theta:[0, \infty) \rightarrow[0, \infty)$ are three functions such that $\varphi$ and $\theta$ are bounded on any bounded interval in $[0, \infty)$ and $\psi$ is continuous. Moreover, assume that

(1) $\psi(x) \leq \varphi(y) \Rightarrow x \leq y$;

(2) for any sequence $\left\{x_{n}\right\}$ in $[0, \infty)$ with $x_{n} \rightarrow t>$ $0, \psi(t)-\limsup _{n \rightarrow \infty} \varphi\left(x_{n}\right)+\liminf _{n \rightarrow \infty} \theta\left(x_{n}\right)>0$.

Then, $T$ has a fixed point.

Proof. Similarly as in the proof of Theorem 6, we construct the sequence $\left\{U_{n}\right\}$ by $U_{0}=U$ and $U_{n}=\operatorname{Conv}\left(T U_{n-1}\right)$ for all $n \geq 1$. If there exists an integer $n_{0} \geq 0$ such that $\mu\left(U_{n_{0}}\right)=$ 0 , then $U_{n_{0}}$ is relatively compact. Hence, from Theorem 2 we conclude that $T$ has a fixed point in $U$. Assume that $\mu\left(U_{n}\right)>0$ for all $n \geq 0$. By applying (12) we get

$$
\begin{aligned}
\psi\left(\mu\left(U_{n+1}\right)\right) & =\psi\left(\mu\left(\operatorname{Conv}\left(T U_{n}\right)\right)\right) \\
& =\psi\left(\mu\left(T U_{n}\right)\right) \leq \varphi\left(\mu\left(U_{n}\right)\right)-\theta\left(\mu\left(U_{n}\right)\right) .
\end{aligned}
$$

Since $\theta \geq 0$, thus from (13) we get $\psi\left(\mu\left(U_{n+1}\right)\right) \leq \varphi\left(\mu\left(U_{n}\right)\right)$, which by condition (1) implies that $\mu\left(U_{n+1}\right) \leq \mu\left(U_{n}\right)$. Hence, $\left\{\mu\left(U_{n}\right\}\right.$ is a decreasing sequence of positive real numbers. So, there exists $r \geq 0$ such that

$$
\lim _{n \rightarrow \infty} \mu\left(U_{n}\right)=r .
$$

We will prove that $\mu\left(U_{n}\right) \rightarrow 0$ as $n \rightarrow \infty$. Taking limit supremum on both sides of (13) and using the properties of the functions $\psi, \varphi$, and $\theta$, we have

$$
\psi(r) \leq \limsup _{n \rightarrow \infty} \varphi\left(\mu U_{n}\right)+\limsup _{n \rightarrow \infty}\left(-\theta\left(\mu U_{n}\right)\right) .
$$

Consequently,

$$
\psi(r) \leq \limsup _{n \rightarrow \infty} \varphi\left(\mu U_{n}\right)-\liminf _{n \rightarrow \infty} \theta\left(\mu U_{n}\right),
$$

which implies that

$$
\psi(r)-\limsup _{n \rightarrow \infty} \varphi\left(\mu U_{n}\right)+\liminf _{n \rightarrow \infty} \theta\left(\mu U_{n}\right) \leq 0 .
$$

So, from condition (2) we conclude that $r=0$. Thus, $\mu\left(U_{n}\right) \rightarrow$ 0 as $n \rightarrow \infty$ and $\lim _{n \rightarrow \infty} \mu\left(U_{n}\right)=0$. Now, since $U_{n+1} \subseteq U_{n}$ 
and $T U_{n} \subseteq U_{n}$, thus from condition (6) of Definition 1 we conclude that $U_{\infty}=\bigcap_{n=1}^{\infty} U_{n}$ is nonempty, closed, convex, and invariant under the operator $T$ and belongs to $\operatorname{Ker} \mu$. Consequently, Theorem 2 implies that $T$ has a fixed point in $U$.

Corollary 10 (Darbo [10]). Let $Q$ be a nonempty, closed, bounded, and convex subset of a Banach space $E$ and let $F$ : $Q \rightarrow Q$ be a continuous mapping. Assume that there exists a constant $k \in[0,1)$ such that $\mu(F X) \leq k \mu(X)$ for any nonempty subset $X$ of Q. Then, $F$ has a fixed point in $Q$.

Proof. Take $\psi, \phi=I$ and $\theta(t)=(1-k) t$ in Theorem 9 .

Taking $\psi=I$ and $\theta=0$ in Theorem 9, we have the following corollary.

Corollary 11. Let $U$ be a nonempty, bounded, closed, and convex subset of the Banach space E. Moreover, assume that $T: U \rightarrow U$ is a continuous function such that

$$
\mu(T X) \leq \varphi(\mu(X))
$$

for any nonempty subset $X$ of $U$, where $\mu$ is an arbitrary measure of noncompactness and $\varphi:[0, \infty) \rightarrow[0, \infty)$ is bounded on any bounded interval in $[0, \infty)$. Moreover, assume that $x \leq \varphi(y) \Rightarrow x \leq y$ and, for any sequence $\left\{x_{n}\right\}$ in $[0, \infty)$ with $x_{n} \rightarrow t>0$,

$$
\limsup _{n \rightarrow \infty} \varphi\left(x_{n}\right)<t
$$

Then, $T$ has a fixed point.

\section{The Solutions of the Integral Equation of Mixed Type}

In this section, we consider the integral equation (5) and prove an existence theorem of solutions of that equation. First, we recall the following two Lemmas of [13] which will be used to prove the existence theorem of the integral equation (5).

Lemma 12. Suppose $x \in C(I)$ and $r: I \rightarrow I$ is a continuous function. Define $(F x)(t)=\max _{[0, r(t)]}|x(\tau)|$ for $t \in I$. Then, $F x \in C(I)$.

Lemma 13. Let $\left(x_{n}\right) \subset C(I)$ and $x \in C(I)$. Suppose that $x_{n} \rightarrow$ $x$ in $C(I)$. Then, $F x_{n} \rightarrow$ Fx uniformly on $I$.

Now, we list the hypotheses which will be used to prove the existence theorem of the integral equation (5).

$\left(H_{1}\right)$ The functions $\alpha_{1}, \alpha_{2}, \gamma_{1}, \gamma_{2}:[0,1] \rightarrow[0,1]$ are continuous.

$\left(H_{2}\right) r:[0,1] \rightarrow[0,1]$ is continuous and nondecreasing on $[0,1]$.
$\left(H_{3}\right) F:[0,1] \times \mathbb{R} \times \mathbb{R} \times \mathbb{R} \rightarrow \mathbb{R}$ is a continuous function and there exist positive real numbers $L_{1}, L_{2}, L_{3}<1$ such that

$$
\begin{aligned}
& \left|F\left(t, x_{1}, x_{2}, x_{3}\right)-F\left(t, y_{1}, y_{2}, y_{3}\right)\right| \\
& \quad \leq L_{1}\left|x_{1}-y_{1}\right|+L_{2}\left|x_{2}-y_{2}\right|+L_{3}\left|x_{3}-y_{3}\right|,
\end{aligned}
$$

for $t \in[0,1]$ and $x_{1}, x_{2}, x_{3}, y_{1}, y_{2}, y_{3} \in \mathbb{R}$. Moreover, assume that $\bar{F}=\sup _{t \in[0,1]}|F(t, 0,0,0)|$.

$\left(H_{4}\right) f:[0,1] \times \mathbb{R} \times \mathbb{R} \rightarrow \mathbb{R}$ is continuous with $f_{0}=\sup \{|f(t, 0,0)| ; t \in[0,1]\}$. Further, there exists continuous and nondecreasing function $\psi_{1}: \mathbb{R}_{+} \rightarrow$ $\mathbb{R}_{+}$with $\psi_{1}(0)=0$ so that

(a) $\left|f\left(t, x_{1}, y_{1}\right)-f\left(t, x_{2}, y_{2}\right)\right| \leq \psi_{1}\left(\left(\left|x_{1}-x_{2}\right|+\mid y_{1}-\right.\right.$ $\left.\left.y_{2} \mid\right) / 2\right)$, for $t \in[0,1]$ and $x_{1}, x_{2}, y_{1}, y_{2} \in \mathbb{R}$.

$\left(H_{5}\right) T: C(I) \rightarrow C(I)$ is a continuous operator such that

$$
\begin{gathered}
\left|(T x)\left(t_{1}\right)-(T x)\left(t_{2}\right)\right| \leq \psi_{1}\left(\left|x\left(t_{2}\right)-x\left(t_{1}\right)\right|\right), \\
|(T x)(t)| \leq a+b\|x\|,
\end{gathered}
$$

for $x \in C(I)$ and $t, t_{1}, t_{2} \in[0,1]$, where $\psi_{1}$ is the existent function in the assumption $\left(H_{4}\right)$ and $a, b$ are positive real numbers.

$\left(H_{6}\right) u:[0,1] \times[0,1] \times \mathbb{R}_{+} \times \mathbb{R}_{+} \rightarrow \mathbb{R}$ is a continuous function and there exists a function $n:[0,1] \rightarrow$ $[0,1]$ being continuous on $[0,1]$ and a function $\phi_{1}$ : $\mathbb{R}_{+} \rightarrow \mathbb{R}_{+}$being continuous and nondecreasing on $\mathbb{R}_{+}$with $\phi_{1}(0)=0$ such that

$$
\begin{aligned}
& \left|u\left(t, s, x_{1}, y_{1}\right)\right| \leq n(t) \phi_{1}\left(\frac{\left|x_{1}-y_{1}\right|}{2}\right), \\
& \left|u\left(t, s, x_{1}, y_{1}\right)-u\left(t, s, x_{2}, y_{2}\right)\right| \\
& \quad \leq n(t) \phi_{1}\left(\frac{\left|x_{1}-x_{2}\right|+\left|y_{1}-y_{2}\right|}{2}\right),
\end{aligned}
$$

for $t, s \in[0,1]$ and $x_{1}, x_{2}, y_{1}, y_{2} \in \mathbb{R}_{+}$.

$\left(H_{7}\right) v:[0,1] \times \mathbb{R}_{+} \times \mathbb{R} \rightarrow \mathbb{R}$ is a continuous function and there exists a continuous function $m: \mathbb{R}_{+} \rightarrow \mathbb{R}_{+}$and a continuous function $k:[0,1] \times \mathbb{R}_{+} \rightarrow \mathbb{R}_{+}$such that $m$ and $s \rightarrow k(t, s)$ are integrable over $\mathbb{R}_{+}$. Moreover, there exists the function $\phi_{2}: \mathbb{R}_{+} \rightarrow \mathbb{R}_{+}$such that $\phi_{2}$ is continuous and nondecreasing with $\phi_{2}(0)=0$ and the following conditions hold.

(a) $\left|v\left(t_{1}, s, x\right)-v\left(t_{2}, s, x\right)\right| \leq m(s) \phi_{2}\left(\left|t_{2}-t_{1}\right|\right)$, $|v(t, s, x)| \leq \phi_{2}(|x|) k(t, s),|v(t, s, x)-v(t, s, y)| \leq$ $\phi_{2}(|x-y|) k(t, s)$, for all $t, t_{1}, t_{2} \in[0,1], s \in \mathbb{R}_{+}$, and $x, y \in \mathbb{R}$.

(b) $\phi_{2}(t)+\psi_{1}(t) \leq \phi(t)$ for all $t \geq 0$, where $\psi_{1}$ and $\phi$ are the existent functions in the assumption $\left(H_{4}\right)$ and Corollary 7, respectively. Moreover, assume that $M=\sup _{t \in[0,1]} \int_{0}^{\infty} k(t, s) d s$. 
$\left(H_{8}\right)$ There exists a positive solution $r_{0}$ such that

$$
\begin{gathered}
L_{1} \psi_{1}\left(r_{0}\right)+L_{2} \bar{n} \frac{\left(a+b r_{0}\right)}{\Gamma(\alpha+1)} \phi_{1}\left(r_{0}\right) \\
+L_{3} M \phi_{2}\left(r_{0}\right)+L_{1} f_{0}+\bar{F} \leq r_{0}, \\
L_{3} M \leq L_{1}+L_{2} \phi_{1}\left(r_{0}\right) \times \frac{1}{\Gamma(\alpha+1)} \leq 1,
\end{gathered}
$$

where $f_{0}=\sup \{f(t, 0,0) ; t \in[0,1]\}$ and $\bar{n}=$ $\sup \left\{n(t) t^{\alpha} ; t \in[0,1]\right\}$.

Now, we can present and prove the main result of this section.

Theorem 14. Under the assumptions $\left(H_{1}\right)-\left(H_{8}\right)$, (5) has at least one solution $x=x(t)$ belonging to the Banach space $C(I)$.

Proof. Define the operator $G$ on the Banach space $C(I)$ by the formula

$$
\begin{gathered}
(G x)(t)=F\left(t, f\left(t, x\left(\alpha_{1}(t)\right), x\left(\alpha_{2}(t)\right)\right), \frac{(T x)(t)}{\Gamma(\alpha)}\right. \\
\times \int_{0}^{t} u\left(t, s, \max _{[0, r(s)]}\left|x\left(\gamma_{1}(\tau)\right)\right|,\right. \\
\left.\quad \max _{[0, r(s)]}\left|x\left(\gamma_{2}(\tau)\right)\right|\right) \\
\quad \times\left((t-s)^{1-\alpha}\right)^{-1} d s \\
\left.\int_{0}^{\infty} v(t, s, x(t)) d s\right), \\
0<\alpha \leq 1, \quad t \in[0,1] .
\end{gathered}
$$

Using the imposed assumptions we infer that $G x$ is continuous on $I$ for each $x \in C(I)$. Moreover, by using our assumptions, for any $t \in I$, we get

$|(G x)(t)|$

$$
\begin{gathered}
=\mid F\left(t, f\left(t, x\left(\alpha_{1}(t)\right), x\left(\alpha_{2}(t)\right)\right),\right. \\
\frac{(T x)(t)}{\Gamma(\alpha)} \int_{0}^{t} u\left(t, s, \max _{[0, r(s)]}\left|x\left(\gamma_{1}(\tau)\right)\right|,\right. \\
\left.\max _{[0, r(s)]}\left|x\left(\gamma_{2}(\tau)\right)\right|\right) \\
\times\left((t-s)^{1-\alpha}\right)^{-1} d s, \\
\left.\int_{0}^{\infty} v(t, s, x(t)) d s\right) \mid
\end{gathered}
$$

$$
\begin{aligned}
& \leq \mid F\left(t, f\left(t, x\left(\alpha_{1}(t)\right), x\left(\alpha_{2}(t)\right)\right),\right. \\
& \frac{(T x)(t)}{\Gamma(\alpha)} \int_{0}^{t} u\left(t, s, \max _{[0, r(s)]}\left|x\left(\gamma_{1}(\tau)\right)\right|,\right. \\
& \left.\max _{[0, r(s)]}\left|x\left(\gamma_{2}(\tau)\right)\right|\right) \\
& \times\left((t-s)^{1-\alpha}\right)^{-1} d s, \\
& \left.\int_{0}^{\infty} v(t, s, x(t)) d s\right)-F(t, 0,0,0) \\
& +|F(t, 0,0,0)| \\
& \leq L_{1}\left|f\left(t, x\left(\alpha_{1}(t)\right), x\left(\alpha_{2}(t)\right)\right)-f(t, 0,0)\right| \\
& +L_{1}|f(t, 0,0)| \\
& +L_{2} \mid \frac{(T x)(t)}{\Gamma(\alpha)} \int_{0}^{t} u\left(t, s, \max _{[0, r(s)]}\left|x\left(\gamma_{1}(\tau)\right)\right|,\right. \\
& \left.\max _{[0, r(s)]}\left|x\left(\gamma_{2}(\tau)\right)\right|\right) \\
& \times\left((t-s)^{1-\alpha}\right)^{-1} d s \\
& +L_{3}\left|\int_{0}^{\infty} v(t, s, x(t)) d s\right|+\bar{F} \\
& \leq L_{1} \psi_{1}\left(\frac{\left|x\left(\alpha_{1}(t)\right)\right|+\left|x\left(\alpha_{2}(t)\right)\right|}{2}\right)+L_{2} \frac{(a+b\|x\|)}{\Gamma(\alpha)} n(t) \\
& \times \int_{0}^{t} \phi_{1}\left(\left|\max _{[0, r(s)]}\right| x\left(\gamma_{2}(\tau)\right) \mid\right. \\
& \left.-\max _{[0, r(s)]}\left|x\left(\gamma_{1}(\tau)\right)\right| \mid \frac{1}{2}\right) \\
& \times(t-s)^{\alpha-1} d s \\
& +L_{3} \int_{0}^{\infty} \phi_{2}(|x(t)|) k(t, s) d s+\bar{F}+L_{1} f_{0} .
\end{aligned}
$$

Since $\psi_{1}, \phi_{1}$, and $\phi_{2}$ are nondecreasing, then from (25) we conclude that

$$
\begin{gathered}
\|(G x)(t)\| \leq L_{1} \psi_{1}(\|x\|)+L_{2} \bar{n} \frac{(a+b\|x\|)}{\Gamma(\alpha+1)} \phi_{1}(\|x\|) \\
+L_{3} M \phi_{2}(\|x\|)+\bar{F}+L_{1} f_{0} .
\end{gathered}
$$

So, in view of assumption $\left(H_{8}\right)$, the operator $G$ transforms $B_{r_{0}}$ into itself. Next, we show that $G$ is continuous on the ball $B_{r_{0}}$. To do this, assume that $\left\{x_{n}\right\}$ is a sequence in $B_{r_{0}}$ such that $x_{n} \rightarrow x$ and show that $G x_{n} \rightarrow G x$. Indeed, for each $t \in I$, we have

$$
\begin{aligned}
& \left|\left(G x_{n}\right)(t)-(G x)(t)\right| \\
& \quad=\mid F\left(t, f\left(t, x_{n}\left(\alpha_{1}(t)\right), x_{n}\left(\alpha_{2}(t)\right)\right),\right.
\end{aligned}
$$




$$
\begin{aligned}
& \frac{\left(T x_{n}\right)(t)}{\Gamma(\alpha)} \int_{0}^{t} u\left(t, s, \max _{[0, r(s)]}\left|x_{n}\left(\gamma_{1}(\tau)\right)\right|,\right. \\
& \left.\max _{[0, r(s)]}\left|x_{n}\left(\gamma_{2}(\tau)\right)\right|\right) \\
& \times\left((t-s)^{1-\alpha}\right)^{-1} d s, \\
& \left.\int_{0}^{\infty} v\left(t, s, x_{n}(t)\right) d s\right) \\
& -F\left(t, f\left(t, x\left(\alpha_{1}(t)\right), x\left(\alpha_{2}(t)\right)\right),\right. \\
& \frac{(T x)(t)}{\Gamma(\alpha)} \int_{0}^{t} u\left(t, s, \max _{[0, r(s)]}\left|x\left(\gamma_{1}(\tau)\right)\right|,\right. \\
& \left.\max _{[0, r(s)]}\left|x\left(\gamma_{2}(\tau)\right)\right|\right) \\
& \times\left((t-s)^{1-\alpha}\right)^{-1} d s \text {, } \\
& \left.\int_{0}^{\infty} v(t, s, x(t)) d s\right) \\
& \leq L_{1} \mid f\left(t, x_{n}\left(\alpha_{1}(t)\right), x_{n}\left(\alpha_{2}(t)\right)\right) \\
& -f\left(t, x\left(\alpha_{1}(t)\right), x\left(\alpha_{2}(t)\right)\right) \\
& +L_{2} \mid \frac{\left(T x_{n}\right)(t)}{\Gamma(\alpha)} \int_{0}^{t} u\left(t, s, \max _{[0, r(s)]}\left|x_{n}\left(\gamma_{1}(\tau)\right)\right|,\right. \\
& \left.\max _{[0, r(s)]}\left|x_{n}\left(\gamma_{2}(\tau)\right)\right|\right) \\
& \times\left((t-s)^{1-\alpha}\right)^{-1} d s \\
& -\frac{(T x)(t)}{\Gamma(\alpha)} \int_{0}^{t} u\left(t, s, \max _{[0, r(s)]}\left|x\left(\gamma_{1}(\tau)\right)\right|,\right. \\
& \left.\max _{[0, r(s)]}\left|x\left(\gamma_{2}(\tau)\right)\right|\right) \\
& \times\left((t-s)^{1-\alpha}\right)^{-1} d s \\
& +L_{3}\left|\int_{0}^{\infty} v\left(t, s, x_{n}(t)\right) d s-\int_{0}^{\infty} v(t, s, x(t)) d s\right| .
\end{aligned}
$$

On the other hand, we have

$$
\begin{gathered}
\mid \frac{\left(T x_{n}\right)(t)}{\Gamma(\alpha)} \int_{0}^{t} u\left(t, s, \max _{[0, r(s)]}\left|x_{n}\left(\gamma_{1}(\tau)\right)\right|\right. \\
\left.\max _{[0, r(s)]}\left|x_{n}\left(\gamma_{2}(\tau)\right)\right|\right) \\
\times\left((t-s)^{1-\alpha}\right)^{-1} d s \\
-\frac{(T x)(t)}{\Gamma(\alpha)} \int_{0}^{t} u\left(t, s, \max _{[0, r(s)]}\left|x\left(\gamma_{1}(\tau)\right)\right|,\right. \\
\times\left((t-s)^{1-\alpha}\right)^{-1} d s \mid
\end{gathered}
$$

$$
\begin{gathered}
\leq \mid \frac{\left(T x_{n}\right)(t)}{\Gamma(\alpha)} \int_{0}^{t} u\left(t, s, \max _{[0, r(s)]}\left|x_{n}\left(\gamma_{1}(\tau)\right)\right|\right. \\
\left.\max _{[0, r(s)]}\left|x_{n}\left(\gamma_{2}(\tau)\right)\right|\right) \\
\times\left((t-s)^{1-\alpha}\right)^{-1} d s
\end{gathered}
$$$$
-\frac{\left(T x_{n}\right)(t)}{\Gamma(\alpha)} \int_{0}^{t} u\left(t, s, \max _{[0, r(s)]}\left|x\left(\gamma_{1}(\tau)\right)\right|\right. \text {, }
$$$$
\left.\max _{[0, r(s)]}\left|x\left(\gamma_{2}(\tau)\right)\right|\right)
$$$$
\times\left((t-s)^{1-\alpha}\right)^{-1} d s
$$$$
+\mid \frac{\left(T x_{n}\right)(t)}{\Gamma(\alpha)} \int_{0}^{t} u\left(t, s, \max _{[0, r(s)]}\left|x\left(\gamma_{1}(\tau)\right)\right|\right. \text {, }
$$$$
\left.\max _{[0, r(s)]}\left|x\left(\gamma_{2}(\tau)\right)\right|\right)
$$$$
\times\left((t-s)^{1-\alpha}\right)^{-1} d s
$$$$
-\frac{(T x)(t)}{\Gamma(\alpha)} \int_{0}^{t} u\left(t, s, \max _{[0, r(s)]}\left|x\left(\gamma_{1}(\tau)\right)\right|,\right.
$$$$
\left.\max _{[0, r(s)]}\left|x\left(\gamma_{2}(\tau)\right)\right|\right)
$$$$
\times\left((t-s)^{1-\alpha}\right)^{-1} d s
$$$$
\leq \frac{\left(a+b\left\|x_{n}\right\|\right)}{\Gamma(\alpha+1)} t^{\alpha} n(t) \phi_{1}
$$$$
\times\left(\frac { 1 } { 2 } \left[\left|\max _{[0, r(s)]}\right| x_{n}\left(\gamma_{1}(\tau)\right) \mid\right.\right.
$$$$
-\max _{[0, r(s)]}\left|x\left(\gamma_{1}(\tau)\right)\right| \mid
$$$$
+\left|\max _{[0, r(s)]}\right| x_{n}\left(\gamma_{2}(\tau)\right) \mid
$$$$
\left.\left.-\max _{[0, r(s)]}\left|x\left(\gamma_{2}(\tau)\right)\right| \mid\right]\right)
$$$$
+\frac{\left\|T x_{n}-T x\right\|}{\Gamma(\alpha+1)} n(t) t^{\alpha} \phi_{1}\left(\left|\max _{[0, r(s)]}\right| x\left(\gamma_{1}(\tau)\right) \mid\right.
$$

$$
\left.-\max _{[0, r(s)]}\left|x\left(\gamma_{2}(\tau)\right)\right| \mid \frac{1}{2}\right) .
$$

Due to (27), (28), and our assumptions, we derive that

$$
\begin{aligned}
\mid\left(G x_{n}\right)(t)- & (G x)(t) \mid \\
\leq L_{1} \psi_{1}( & \left(\left|x_{n}\left(\alpha_{1}(t)\right)-x\left(\alpha_{1}(t)\right)\right|\right. \\
& \left.\left.\quad+\left|x_{n}\left(\alpha_{2}(t)\right)-x\left(\alpha_{2}(t)\right)\right|\right) \frac{1}{2}\right)
\end{aligned}
$$




$$
\begin{aligned}
& +L_{2} \frac{\left(a+b\left\|x_{n}\right\|\right)}{\Gamma(\alpha+1)} t^{\alpha} n(t) \phi_{1} \\
& \times\left(\left|\max _{[0, r(s)]}\right| x_{n}\left(\gamma_{1}(\tau)\right) \mid\right. \\
& -\max _{[0, r(s)]} \mid x\left(\gamma_{1}(\tau)\right) \| \\
& +\left|\max _{[0, r(s)]}\right| x_{n}\left(\gamma_{2}(\tau)\right) \mid \\
& \left.\quad-\max _{[0, r(s)]} \mid x\left(\gamma_{2}(\tau)\right) \|\right) \\
& +\frac{\left\|T x_{n}-T x\right\|}{\Gamma(\alpha+1)} n(t) t^{\alpha} \phi_{1} \\
& \times\left(\left|\max _{[0, r(s)]}\right| x\left(\gamma_{2}(\tau)\right) \mid\right. \\
& \quad-\max _{[0, r(s)]}\left|x\left(\gamma_{1}(\tau)\right)\right| \\
& \left.\quad-\max _{[0, r(s)]} \mid x\left(\gamma_{1}(\tau)\right) \|\right) \\
& +L_{3} \phi_{2}\left(\left|x_{n}(t)-x(t)\right|\right) \int_{0}^{\infty} k(t, s) d s .
\end{aligned}
$$

Using (29), Lemma 13, and the imposed assumptions, we have $\left\|G x_{n}-G x\right\| \rightarrow 0$ as $n \rightarrow \infty$.

Now, let $X$ be a nonempty subset of the ball $B_{r_{0}}, x \in X$, and fix arbitrarily $\varepsilon>0$. Choose $t_{1}, t_{2} \in[0,1]$ such that $t_{1}<t_{2}$ and $t_{2}-t_{1} \leq \varepsilon$. Then, taking into account our assumptions, we have

$$
\begin{gathered}
\left|(G x)\left(t_{2}\right)-(G x)\left(t_{1}\right)\right| \\
=\mid F\left(t_{2}, f\left(t_{2}, x\left(\alpha_{1}\left(t_{2}\right)\right), x\left(\alpha_{2}\left(t_{2}\right)\right)\right),\right. \\
\frac{(T x)\left(t_{2}\right)}{\Gamma(\alpha)} \int_{0}^{t_{2}} u\left(t_{2}, s, \max _{[0, r(s)]}\left|x\left(\gamma_{1}(\tau)\right)\right|,\right. \\
\left.\max _{[0, r(s)]}\left|x\left(\gamma_{2}(\tau)\right)\right|\right) \\
\times\left(\left(t_{2}-s\right)^{1-\alpha}\right)^{-1} d s \\
\left.\int_{0}^{\infty} v\left(t_{2}, s, x\left(t_{2}\right)\right) d s\right) \\
-F\left(t_{1}, f\left(t_{1}, x\left(\alpha_{1}\left(t_{1}\right)\right), x\left(\alpha_{2}\left(t_{1}\right)\right)\right),\right. \\
\frac{(T x)\left(t_{1}\right)}{\Gamma(\alpha)} \int_{0}^{t_{1}} u\left(t_{1}, s, \max _{[0, r(s)]}\left|x\left(\gamma_{1}(\tau)\right)\right|,\right. \\
\left.\max _{[0, r(s)]}\left|x\left(\gamma_{2}(\tau)\right)\right|\right) \\
\quad \times\left(\left(t_{1}-s\right)^{1-\alpha}\right)^{-1} d s, \\
\left.\int_{0}^{\infty} v\left(t_{1}, s, x\left(t_{1}\right)\right) d s\right) \mid
\end{gathered}
$$

$$
\begin{gathered}
\leq L_{1} \mid f\left(t_{2}, x\left(\alpha_{1}\left(t_{2}\right)\right), x\left(\alpha_{2}\left(t_{2}\right)\right)\right) \\
-f\left(t_{2}, x\left(\alpha_{1}\left(t_{1}\right)\right), x\left(\alpha_{2}\left(t_{1}\right)\right)\right) \mid \\
+L_{1} \mid f\left(t_{2}, x\left(\alpha_{1}\left(t_{1}\right)\right), x\left(\alpha_{2}\left(t_{1}\right)\right)\right) \\
-f\left(t_{1}, x\left(\alpha_{1}\left(t_{1}\right)\right), x\left(\alpha_{2}\left(t_{1}\right)\right)\right) \mid \\
+L_{2} \mid \frac{(T x)\left(t_{2}\right)}{\Gamma(\alpha)} \int_{0}^{t_{2}} u\left(t_{2}, s, \max _{[0, r(s)]}\left|x\left(\gamma_{1}(\tau)\right)\right|,\right. \\
\left.\quad \max _{[0, r(s)]}\left|x\left(\gamma_{2}(\tau)\right)\right|\right) \\
\quad \times \frac{(T x)\left(t_{1}\right)}{\Gamma(\alpha)} \int_{0}^{t_{1}} u\left(t_{1}, s, \max _{[0, r(s)]}\left|x\left(\gamma_{1}(\tau)\right)\right|,\right. \\
\left.\max _{[0, r(s)]}\left|x\left(\gamma_{2}(\tau)\right)\right|\right) \\
\times\left(\left(t_{1}-s\right)^{1-\alpha}\right)^{-1} d s \mid \\
+L_{3} \int_{0}^{\infty}\left|v\left(t_{2}, s, x\left(t_{2}\right)\right)-v\left(t_{1}, s, x\left(t_{2}\right)\right)\right| d s \\
+L_{3} \int_{0}^{\infty}\left|v\left(t_{1}, s, x\left(t_{2}\right)\right)-v\left(t_{1}, s, x\left(t_{1}\right)\right)\right| d s .
\end{gathered}
$$

Moreover, we have

$$
\begin{gathered}
\mid \frac{(T x)\left(t_{2}\right)}{\Gamma(\alpha)} \int_{0}^{t_{2}} u\left(t_{2}, s, \max _{[0, r(s)]}\left|x\left(\gamma_{1}(\tau)\right)\right|,\right. \\
\left.\max _{[0, r(s)]}\left|x\left(\gamma_{2}(\tau)\right)\right|\right) \\
\times\left(\left(t_{2}-s\right)^{1-\alpha}\right)^{-1} d s \\
-\frac{(T x)\left(t_{1}\right)}{\Gamma(\alpha)} \int_{0}^{t_{1}} u\left(t_{1}, s, \max _{[0, r(s)]}\left|x\left(\gamma_{1}(\tau)\right)\right|,\right. \\
\quad \times\left(\left(t_{1}-s\right)^{1-\alpha}\right)^{-1} d s \mid \\
\leq \mid \frac{(T x)\left(t_{2}\right)}{\Gamma(\alpha)} \int_{0}^{t_{2}} u\left(t_{2}, s, \max _{[0, r(s)]}\left|x\left(\gamma_{1}(\tau)\right)\right|,\right. \\
\left.\quad \max _{[0, r(s)]}\left|x\left(\gamma_{2}(\tau)\right)\right|\right) \\
\quad \times\left(\left(t_{2}-s\right)^{1-\alpha}\right)^{-1} d s \\
-\frac{(T x)\left(t_{2}\right)}{\Gamma(\alpha)} \int_{0}^{t_{1}} u\left(t_{1}, s, \max _{[0, r(s)]}\left|x\left(\gamma_{1}(\tau)\right)\right|,\right. \\
\left.\max _{[0, r(s)]}\left|x\left(\gamma_{2}(\tau)\right)\right|\right)
\end{gathered}
$$




$$
\begin{aligned}
& \times\left(\left(t_{1}-s\right)^{1-\alpha}\right)^{-1} d s \\
& +\mid \frac{(T x)\left(t_{2}\right)}{\Gamma(\alpha)} \int_{0}^{t_{1}} u\left(t_{1}, s, \max _{[0, r(s)]}\left|x\left(\gamma_{1}(\tau)\right)\right|\right. \\
& \left.\max _{[0, r(s)]}\left|x\left(\gamma_{2}(\tau)\right)\right|\right) \\
& \times\left(\left(t_{1}-s\right)^{1-\alpha}\right)^{-1} d s \\
& -\frac{(T x)\left(t_{1}\right)}{\Gamma(\alpha)} \int_{0}^{t_{1}} u\left(t_{1}, s, \max _{[0, r(s)]}\left|x\left(\gamma_{1}(\tau)\right)\right|\right. \\
& \left.\max _{[0, r(s)]}\left|x\left(\gamma_{2}(\tau)\right)\right|\right) \\
& \times\left(\left(t_{1}-s\right)^{1-\alpha}\right)^{-1} d s \\
& \leq \frac{(T x)\left(t_{2}\right)}{\Gamma(\alpha)}\left[\mid \int_{0}^{t_{2}} u\left(t_{2}, s, \max _{[0, r(s)]}\left|x\left(\gamma_{1}(\tau)\right)\right|,\right.\right. \\
& \left.\max _{[0, r(s)]}\left|x\left(\gamma_{2}(\tau)\right)\right|\right) \\
& \times\left(\left(t_{2}-s\right)^{1-\alpha}\right)^{-1} d s \\
& -\int_{0}^{t_{1}} u\left(t_{2}, s, \max _{[0, r(s)]}\left|x\left(\gamma_{1}(\tau)\right)\right|,\right. \\
& \left.\max _{[0, r(s)]}\left|x\left(\gamma_{2}(\tau)\right)\right|\right) \\
& \times\left(\left(t_{2}-s\right)^{1-\alpha}\right)^{-1} d s \\
& +\mid \int_{0}^{t_{1}} u\left(t_{2}, s, \max _{[0, r(s)]}\left|x\left(\gamma_{1}(\tau)\right)\right|\right. \\
& \left.\max _{[0, r(s)]}\left|x\left(\gamma_{2}(\tau)\right)\right|\right) \\
& \times\left(\left(t_{2}-s\right)^{1-\alpha}\right)^{-1} d s \\
& -\int_{0}^{t_{1}} u\left(t_{1}, s, \max _{[0, r(s)]}\left|x\left(\gamma_{1}(\tau)\right)\right|,\right. \\
& \left.\max _{[0, r(s)]}\left|x\left(\gamma_{2}(\tau)\right)\right|\right) \\
& \times\left(\left(t_{2}-s\right)^{1-\alpha}\right)^{-1} d s \\
& +\mid \int_{0}^{t_{1}} u\left(t_{1}, s, \max _{[0, r(s)]}\left|x\left(\gamma_{1}(\tau)\right)\right|\right. \\
& \left.\max _{[0, r(s)]}\left|x\left(\gamma_{2}(\tau)\right)\right|\right) \\
& \times\left(\left(t_{2}-s\right)^{1-\alpha}\right)^{-1} d s \\
& -\int_{0}^{t_{1}} u\left(t_{1}, s, \max _{[0, r(s)]}\left|x\left(\gamma_{1}(\tau)\right)\right|\right. \\
& \left.\max _{[0, r(s)]}\left|x\left(\gamma_{2}(\tau)\right)\right|\right)
\end{aligned}
$$

Thus,

$$
\begin{aligned}
& \mid \frac{(T x)\left(t_{2}\right)}{\Gamma(\alpha)} \int_{0}^{t_{2}} u\left(t_{2}, s, \max _{[0, r(s)]}\left|x\left(\gamma_{1}(\tau)\right)\right|,\right. \\
& \left.\max _{[0, r(s)]}\left|x\left(\gamma_{2}(\tau)\right)\right|\right) \\
& \times\left(\left(t_{2}-s\right)^{1-\alpha}\right)^{-1} d s \\
& -\frac{(T x)\left(t_{1}\right)}{\Gamma(\alpha)} \int_{0}^{t_{1}} u\left(t_{1}, s, \max _{[0, r(s)]}\left|x\left(\gamma_{1}(\tau)\right)\right|,\right. \\
& \left.\max _{[0, r(s)]}\left|x\left(\gamma_{2}(\tau)\right)\right|\right) \\
& \times\left(\left(t_{1}-s\right)^{1-\alpha}\right)^{-1} d s \\
& \leq \frac{(a+b\|x\|)}{\Gamma(\alpha)} n\left(t_{2}\right) \phi_{1}(\|x\|) \\
& \times \int_{t_{1}}^{t_{2}}\left(t_{2}-s\right)^{\alpha-1} d s+\omega_{r_{0}}(u, \varepsilon) \int_{0}^{t_{1}}\left(t_{2}-s\right)^{\alpha-1} d s \\
& +n\left(t_{1}\right) \phi_{1}(\|x\|) \int_{0}^{t_{1}}\left\{\left(t_{1}-s\right)^{\alpha-1}-\left(t_{2}-s\right)^{\alpha-1}\right\} d s \\
& +\frac{\psi_{1}(\omega(x, \varepsilon))}{\Gamma(\alpha)} n\left(t_{1}\right) \phi_{1}(\|x\|) \int_{0}^{t_{1}}\left(t_{1}-s\right)^{\alpha-1} d s \\
& =\frac{(a+b\|x\|)}{\Gamma(\alpha+1)} n\left(t_{2}\right) \phi_{1}(\|x\|)\left(t_{2}-t_{1}\right)^{\alpha} \\
& +\omega_{r_{0}}(u, \varepsilon) \times \frac{1}{\alpha}\left(-\left(t_{2}-t_{1}\right)^{\alpha}+t_{2}^{\alpha}\right) \\
& +n\left(t_{1}\right) \phi_{1}(\|x\|)\left(\left(t_{2}-t_{1}\right)^{\alpha}+t_{1}^{\alpha}-t_{2}^{\alpha}\right) \times \frac{1}{\alpha} \\
& +\frac{1}{\Gamma(\alpha+1)} \times \psi_{1}(\omega(x, \varepsilon)) n\left(t_{1}\right) \phi_{1}(\|x\|) \times t_{1}^{\alpha} \\
& \leq \frac{\left(a+b r_{0}\right)}{\Gamma(\alpha+1)} \phi_{1}\left(r_{0}\right) \times \varepsilon^{\alpha}+\omega_{r_{0}}(u, \varepsilon) \times \frac{1}{\alpha} \times-\varepsilon^{\alpha}
\end{aligned}
$$




$$
\begin{aligned}
& +\phi_{1}\left(r_{0}\right) \varepsilon^{\alpha} \times \frac{1}{\alpha} \\
& +\frac{1}{\Gamma(\alpha+1)} \psi_{1}(\omega(x, \varepsilon)) \phi_{1}\left(r_{0}\right) .
\end{aligned}
$$

Using (30), (32), and the assumptions of Theorem 14, we earn

$$
\begin{aligned}
& \left|(G x)\left(t_{2}\right)-(G x)\left(t_{1}\right)\right| \\
& \leq L_{1}\left[\psi_{1}\left(\frac{\omega\left(x, \omega\left(\alpha_{1}, \varepsilon\right)\right)+\omega\left(x, \omega\left(\alpha_{2}, \varepsilon\right)\right)}{2}\right)\right. \\
& \left.+\omega_{r_{0}}(f, \varepsilon)\right] \\
& +L_{2}\left[\frac{\left(a+b r_{0}\right)}{\Gamma(\alpha)} \phi_{1}\left(r_{0}\right) \times \frac{1}{\alpha} \varepsilon^{\alpha}\right. \\
& \left.+\omega_{r_{0}}(u, \varepsilon) \times \frac{-1}{\alpha} \varepsilon^{\alpha}+\phi_{1}\left(r_{0}\right) \varepsilon^{\alpha} \times \frac{1}{\alpha}\right] \\
& +L_{2} \times \frac{1}{\Gamma(\alpha+1)} \psi_{1}(\omega(x, \varepsilon)) \phi_{1}\left(r_{0}\right) \\
& +L_{3} \phi_{2}(\varepsilon) \int_{0}^{\infty} m(s) d s \\
& +L_{3} \phi_{2}\left(\left|x\left(t_{2}\right)-x\left(t_{1}\right)\right|\right) \int_{0}^{\infty} k\left(t_{1}, s\right) d s
\end{aligned}
$$

where

$$
\begin{array}{r}
\omega_{r_{0}}(u, \varepsilon)=\sup \left\{\left|u\left(t, s, x_{1}, x_{2}\right)-u\left(\tau, s, x_{1}, x_{2}\right)\right| ;\right. \\
t, s, \tau \in[0,1], \\
\left.x_{1}, x_{2} \in\left[0, r_{0}\right],|t-\tau| \leq \varepsilon\right\}, \\
\omega_{r_{0}}(f, \varepsilon)=\sup \left\{\left|f\left(t_{2}, x, y\right)-f\left(t_{1}, x, y\right)\right|:\right. \\
t_{1}, t_{2} \in[0,1], \\
\left.\left|t_{2}-t_{1}\right|<\varepsilon, x, y \in\left[-r_{0}, r_{0}\right]\right\} .
\end{array}
$$

The estimate (33) implies that

$\omega(G x, \varepsilon)$

$$
\begin{aligned}
& \leq L_{1} \psi_{1}\left(\frac{\omega\left(x, \omega\left(\alpha_{1}, \varepsilon\right)\right)+\omega\left(x, \omega\left(\alpha_{2}, \varepsilon\right)\right)}{2}\right) \\
&+ L_{1} \omega_{r_{0}}(f, \varepsilon) \\
&+L_{2}\left[\frac{\left(a+b r_{0}\right)}{\Gamma(\alpha+1)} \phi_{1}\left(r_{0}\right) \times \frac{1}{\alpha} \varepsilon^{\alpha}\right. \\
&\left.+\omega_{r_{0}}(u, \varepsilon) \times \frac{-1}{\alpha} \varepsilon^{\alpha}+\phi_{1}\left(r_{0}\right) \varepsilon^{\alpha} \times \frac{1}{\alpha}\right]
\end{aligned}
$$

$$
\begin{aligned}
& +L_{2} \times \frac{1}{\Gamma(\alpha+1)} \psi_{1}(\omega(x, \varepsilon)) \phi_{1}\left(r_{0}\right) \\
& +L_{3} \int_{0}^{\infty} \phi_{2}(\varepsilon) m(s) d s+L_{3} \phi_{2}(\omega(x, \varepsilon)) M .
\end{aligned}
$$

It follows from assumptions $\left(H_{1}\right),\left(H_{4}\right)$, and $\left(H_{6}\right)$ that the functions $\alpha_{1}, \alpha_{2}, f$, and $u$ are uniformly continuous on the sets $[0,1],[0,1],[0,1] \times\left[-r_{0}, r_{0}\right] \times\left[-r_{0}, r_{0}\right]$, and $[0,1] \times[0,1] \times$ $\left[0, r_{0}\right] \times\left[0, r_{0}\right]$, respectively. Consequently, we infer that

$$
\begin{aligned}
\lim _{\varepsilon \rightarrow 0} \omega\left(\alpha_{1}, \varepsilon\right) & =\lim _{\varepsilon \rightarrow 0} \omega\left(\alpha_{2}, \varepsilon\right) \\
& =\lim _{\varepsilon \rightarrow 0} \omega_{r_{0}}(f, \varepsilon)=\lim _{\varepsilon \rightarrow 0} \omega_{r_{0}}(u, \varepsilon)=0 .
\end{aligned}
$$

Hence, using assumptions $\left(H_{4}\right)$ and $\left(H_{7}\right)$ and estimate (35), we get

$\omega_{0}(G X)$

$$
\begin{gathered}
\leq L_{1} \lim _{\varepsilon \rightarrow 0} \psi_{1}\left(\frac{\omega\left(X, \omega\left(\alpha_{1}, \varepsilon\right)\right)+\omega\left(X, \omega\left(\alpha_{2}, \varepsilon\right)\right)}{2}\right) \\
+\lim _{\varepsilon \rightarrow 0}\left(L_{2} \times \frac{1}{\Gamma(\alpha+1)} \psi_{1}(\omega(X, \varepsilon)) \phi_{1}\left(r_{0}\right)\right. \\
\left.+L_{3} M \phi_{2}(\omega(X, \varepsilon))\right) .
\end{gathered}
$$

Since the functions $\psi_{1}$ and $\phi_{2}$ are continuous and $\psi_{1}(t)+$ $\phi_{2}(t) \leq \phi(t)$, then from (36), (37), and the assumption $\left(H_{8}\right)$, we conclude that

$$
\begin{aligned}
\omega_{0}(G X) \leq & L_{1} \psi_{1}\left(\omega_{0}(X)\right) \\
& +L_{2} \times \frac{1}{\Gamma(\alpha+1)} \phi_{1}\left(r_{0}\right) \times \psi_{1}\left(\omega_{0}(X)\right) \\
& +L_{3} M \phi_{2}\left(\omega_{0}(X)\right) \\
\leq & \left(L_{1}+L_{2} \phi_{1}\left(r_{0}\right) \times \frac{1}{\Gamma(\alpha+1)}\right) \\
& \times\left(\psi_{1}\left(\omega_{0}(X)\right)+\phi_{2}\left(\omega_{0}(X)\right)\right) \\
\leq & \phi\left(\omega_{0}(X)\right) .
\end{aligned}
$$

Thus, Corollary 7 completes the proof.

Now, we present the following example which shows that Theorem 14 can be applied to obtain solutions of the integral equation (39) but the existent results in $[5,6]$ are inapplicable. 
Example 15. Now, we investigate the following integral equation:

$x(t)$

$$
\begin{aligned}
=\frac{e^{-t}}{100} \cos ( & \frac{e^{-t}}{800\left(1+t^{2}\right)(1+|x(t)|+|x(2 t)|)} \\
& +\frac{1}{100} \times \cos \left(\frac{1}{(1+(1 / 8)|x(t)|)}\right) \frac{1}{\Gamma(1 / 2)} \\
& \times \int_{0}^{t}\left(\operatorname { a r c t a n } \left(e^{-t s}\left|\max _{[0, s]}\right| x(\tau) \mid\right.\right. \\
& \times\left((t-s)^{1 / 2}\right)^{-1} d s \\
& \left.+\frac{1}{100} \int_{0}^{\infty} \frac{e^{-s}}{1+t / 8} \sin \left(\frac{|x(t)|}{8}\right) d s\right),
\end{aligned}
$$

for $t \in[0,1]$. Equation (39) is a special case of the integral equation (5), where

$$
\begin{gathered}
F(t, x, y, z)=\frac{e^{-t}}{100} \cos \left(\frac{x+y+z}{100}\right), \\
f(t, x, y)=\frac{e^{-t}}{8\left(1+t^{2}\right)(1+|x|+|y|)}, \\
\alpha_{1}(t)=\gamma_{1}(t)=r(t)=t, \\
\alpha_{2}(t)=\gamma_{2}(t)=2 t, \\
(T x)(t)=\cos \left(\frac{1}{(1+(1 / 8)|x(t)|)}\right), \\
u(t, s, x, y)=\arctan \left(\frac{e^{-t s}|x-y|}{8}\right), \\
v(t, s, x)=\frac{e^{-s}}{1+t / 8} \sin \left(\frac{|x|}{8}\right) .
\end{gathered}
$$

Then, it is easily seen that $\alpha_{1}, \alpha_{2}, \gamma_{1}, \gamma_{2}$, and $r$ satisfy the assumptions $\left(H_{1}\right)$ and $\left(H_{2}\right)$ of the Theorem 14 . Further, the function $F$ satisfies assumption $\left(H_{3}\right)$ with $L_{1}=L_{2}=L_{3}=$ $1 / 10000$ and $\bar{F}=1 / 100$. Since $f(t, x, y)=e^{-t} / 8\left(1+t^{2}\right)(1+$ $|x|+|y|)$, then, for all $t \in[0,1]$ and $x_{1}, x_{2}, y_{1}, y_{2} \in \mathbb{R}$, we have

$$
\begin{aligned}
\left|f\left(t, x_{1}, y_{1}\right)-f\left(t, x_{2}, y_{2}\right)\right| & =\mid \frac{e^{-t}}{8\left(1+t^{2}\right)\left(1+\left|x_{1}\right|+\left|y_{1}\right|\right)} \\
& \quad-\frac{e^{-t}}{8\left(1+t^{2}\right)\left(1+\left|x_{2}\right|+\left|y_{2}\right|\right)} \mid
\end{aligned}
$$

$$
\begin{aligned}
& =\frac{e^{-t}}{8\left(1+t^{2}\right)}\left|\frac{\left|x_{2}\right|+\left|y_{2}\right|-\left|x_{1}\right|-\left|y_{1}\right|}{\left(1+\left|x_{1}\right|+\left|y_{1}\right|\right)\left(1+\left|x_{2}\right|+\left|y_{2}\right|\right)}\right| \\
& \leq \frac{1}{8}\left(\left|x_{2}-x_{1}\right|+\left|y_{2}-y_{1}\right|\right) .
\end{aligned}
$$

So, the assumption $\left(H_{4}\right)$ is satisfied with $\psi_{1}(t)=(1 / 4) t$ and $f_{0}=1 / 8$. In this example, we have $(T x)(t)=\cos (1 /(1+$ $(1 / 8)|x(t)|))$ and this operator satisfies assumption $\left(H_{5}\right)$ with $\psi_{1}(t)=t / 8, a=1$, and $b=0$. On the other hand, for all $t, s \in[0,1]$ and $x, y, x_{1}, x_{2}, y_{1}, y_{2} \in \mathbb{R}_{+}$, we get

$$
\begin{aligned}
|u(t, s, x, y)| & =\left|\arctan \left(e^{-t s} \frac{|x-y|}{8}\right)\right| \\
\leq & e^{-t}\left|\frac{|x-y|}{8}\right|, \\
\left|u\left(t, s, x_{1}, y_{1}\right)-u\left(t, s, x_{2}, y_{2}\right)\right|= & \mid \arctan \left(\frac{e^{-t s}\left|x_{1}-y_{1}\right|}{8}\right) \\
& -\arctan \left(\frac{e^{-t s}\left|x_{2}-y_{2}\right|}{8}\right) \mid \\
\leq & e^{-t}\left|\frac{\left|x_{1}-y_{1}\right|}{8}-\frac{\left|x_{2}-y_{2}\right|}{8}\right| \\
= & e^{-t}\left|\frac{\left|x_{1}-y_{1}\right|-\left|x_{2}-y_{2}\right|}{8}\right| \\
\leq & e^{-t} \frac{\left|x_{1}-x_{2}\right|+\left|y_{1}-y_{2}\right|}{8} .
\end{aligned}
$$

So, $\phi_{1}(t)=(1 / 4) t$ and $n(t)=e^{-t}$. Moreover, $\bar{n}=$ $\sup \left\{\sqrt{t} e^{-t} ; t \in[0,1]\right\}=1 / e$. Thus, assumption $\left(H_{6}\right)$ holds. Now, notice that the function $v(t, s, x)=\left(e^{-s} /(1+\right.$ $t / 8)) \sin (|x| / 8)$ is continuous and satisfies the conditions (a) and (b) of the assumption $\left(H_{7}\right)$. Indeed, for all $t, t_{1}, t_{2} \in[0,1]$, $s \in \mathbb{R}_{+}$, and $x, y \in \mathbb{R}$, we have

$$
\begin{aligned}
& \left|v\left(t_{2}, s, x\right)-v\left(t_{1}, s, x\right)\right| \\
& \quad=\left|\frac{e^{-s}}{1+t_{2} / 8} \sin \left(\frac{|x|}{8}\right)-\frac{e^{-s}}{1+t_{1} / 8} \sin \left(\frac{|x|}{8}\right)\right| \\
& \quad \leq e^{-s} \frac{\left|t_{2}-t_{1}\right|}{8}, \\
& |v(t, s, x)-v(t, s, y)| \\
& \quad=\left|\frac{e^{-s}}{1+t / 8} \sin \left(\frac{|x|}{8}\right)-\frac{e^{-s}}{1+t / 8} \sin \left(\frac{|y|}{8}\right)\right| \\
& \quad \leq \frac{e^{-s}}{1+t / 8}\left(\frac{|x-y|}{8}\right) .
\end{aligned}
$$


So, $\phi_{2}(t)=t / 8, m(s)=e^{-s}$, and $k(t, s)=e^{-s} /(1+t / 8)$. Moreover, $M=\sup _{t \in[0,1]} \int_{0}^{\infty} k(t, s) d s=1, \psi_{1}(t)+\phi_{2}(t)=$ $t / 4=\phi(t)$, and the condition (b) of the assumption $\left(H_{7}\right)$ holds with $\phi(t)=t / 4$. Thus, the existent inequalities in assumption $\left(H_{8}\right)$ have the forms

$$
\begin{aligned}
& \frac{1}{40000} r_{0}+\frac{1}{40000 e} \times \frac{1}{\Gamma(3 / 2)} r_{0}+\frac{1}{10000} \times \frac{r_{0}}{8} \\
& +\frac{1}{8} \times \frac{1}{10000}+\frac{1}{100} \leq r_{0}, \\
& \frac{1}{10000} \leq \frac{1}{10000}+\frac{1}{10000} \times \frac{r_{0}}{4} \times \frac{1}{\Gamma(3 / 2)} \leq 1 .
\end{aligned}
$$

It is easily seen that the last inequalities have a positive solution. For example, $r_{0}=1 / 2$. We see that all assumptions of Theorem 14 are satisfied. Consequently, from Theorem 14 the integral equation (39) has a solution in the space $C(I)$.

\section{Conflict of Interests}

The authors declare that there is no conflict of interests regarding the publication of this paper.

\section{References}

[1] K. Kuratowski, "Sur les espaces complets," Fundamenta Mathematicae, vol. 5, pp. 301-309, 1930.

[2] G. Darbo, "Punti uniti in trasformazioni a codominio non compatto," The Rendiconti del Seminario Matematico della Università di Padova, vol. 24, pp. 84-92, 1955.

[3] A. Aghajani, J. Banaś, and N. Sabzali, "Some generalizations of Darbo fixed point theorem and applications," Bulletin of the Belgian Mathematical Society, vol. 20, no. 2, pp. 345-358, 2013.

[4] A. Aghajani, R. Allahyari, and M. Mursaleen, "A generalization of Darbo's theorem with application to the solvability of systems of integral equations," Journal of Computational and Applied Mathematics, vol. 260, pp. 68-77, 2014.

[5] M. A. Darwish and K. Sadarangani, "Nondecreasing solutions of a quadratic Abel equation with supremum in the kernel," Applied Mathematics and Computation, vol. 219, no. 14, pp. 7830-7836, 2013.

[6] Z. Xia, "Global asymptotic stability for nonlinear functional integral equation of mixed type," Journal of Applied Mathematics, vol. 2013, Article ID 193602, 11 pages, 2013.

[7] X. Huang and J. Cao, "On attractivity and positivity of solutions for functional integral equations of fractional order," Mathematical Problems in Engineering, vol. 2013, Article ID 916369, 17 pages, 2013.

[8] B. S. Choudhury, N. Metiya, and M. Posttolache, "A generalized weak contraction principle with applications to coupled coincidence point problems," Fixed Point Theory and Applications, vol. 2013, article 152, 2013.

[9] M. Berzig, "Generalization of the Banach contraction principle," 2013.

[10] J. Banaś and K. Goebel, Measures of Noncompactness in Banach Spaces, vol. 60 of Lecture Notes in Pure and Applied Mathematics, Marcel Dekker, New York, NY, USA, 1980.

[11] J. Banas and M. Lecko, "Fixed points of the product of operators in Banach algebra," Panamerican Mathematical Journal, vol. 12, no. 2, pp. 101-109, 2002.
[12] R. Agarwal, M. Meehan, and D. O'Regan, Fixed Point Theory and Applications, Cambridge University Press, Cambridge, UK, 2004.

[13] J. Caballero, B. López, and K. Sadarangani, "On monotonic solutions of an integral equation of Volterra type with supremum," Journal of Mathematical Analysis and Applications, vol. 305, no. 1, pp. 304-315, 2005. 


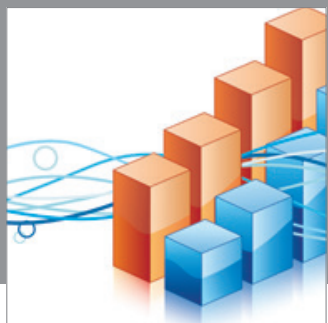

Advances in

Operations Research

mansans

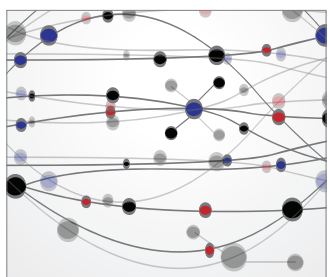

The Scientific World Journal
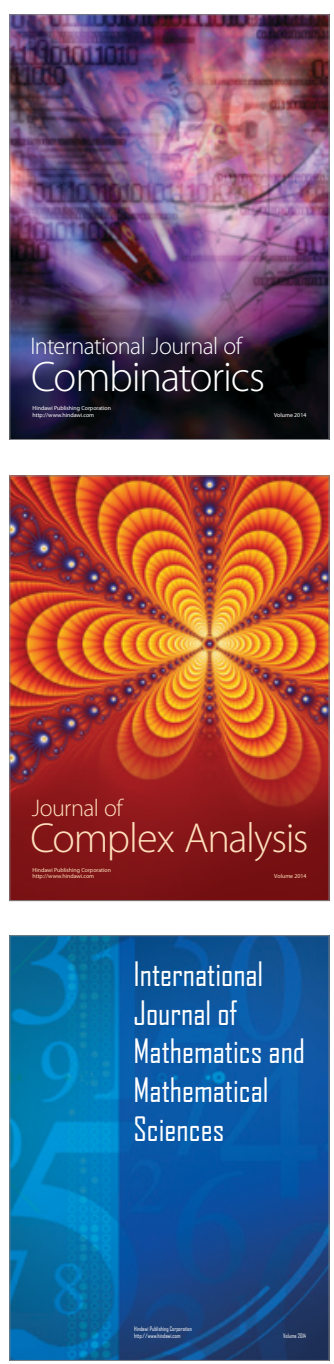
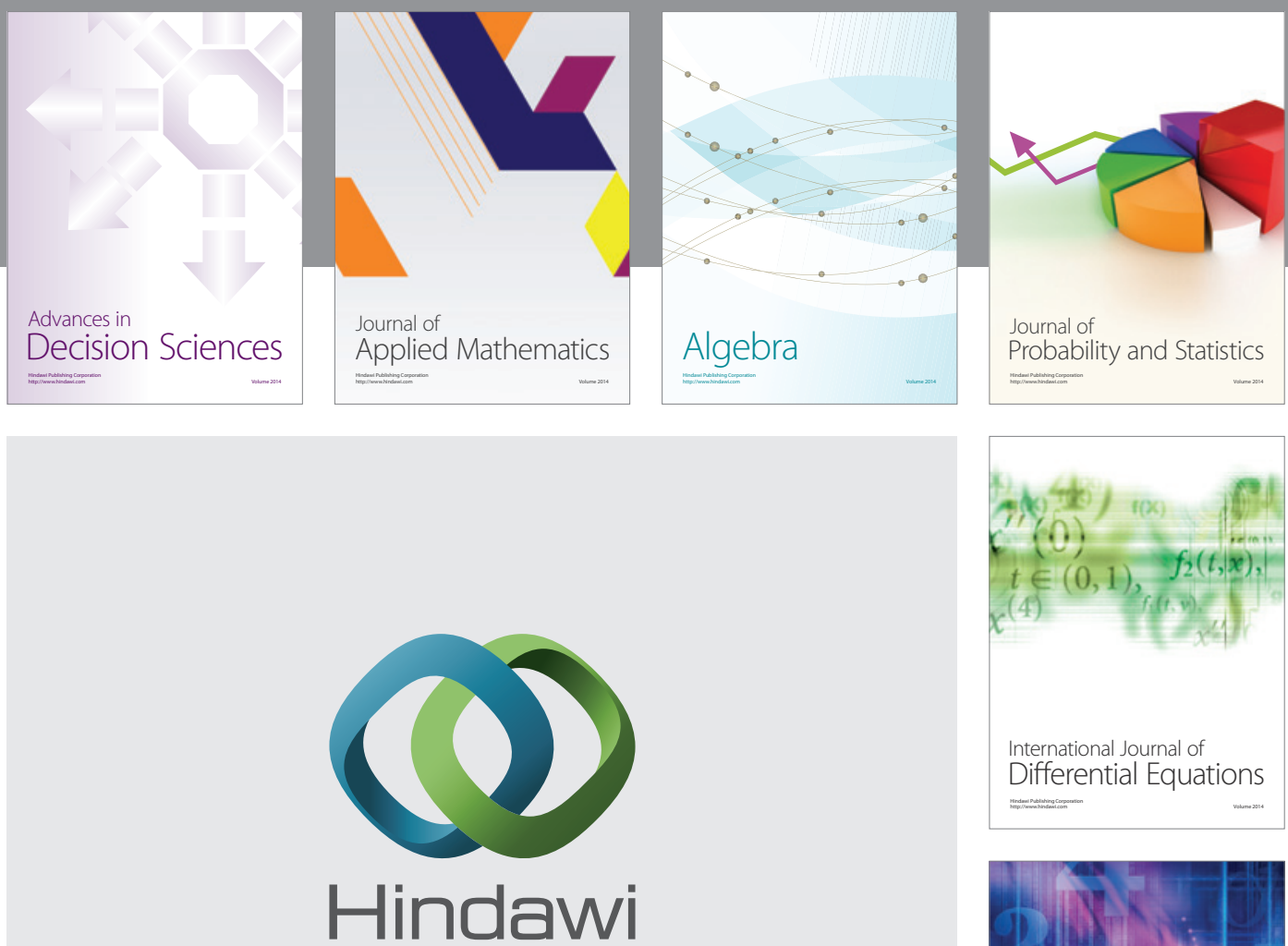

Submit your manuscripts at http://www.hindawi.com
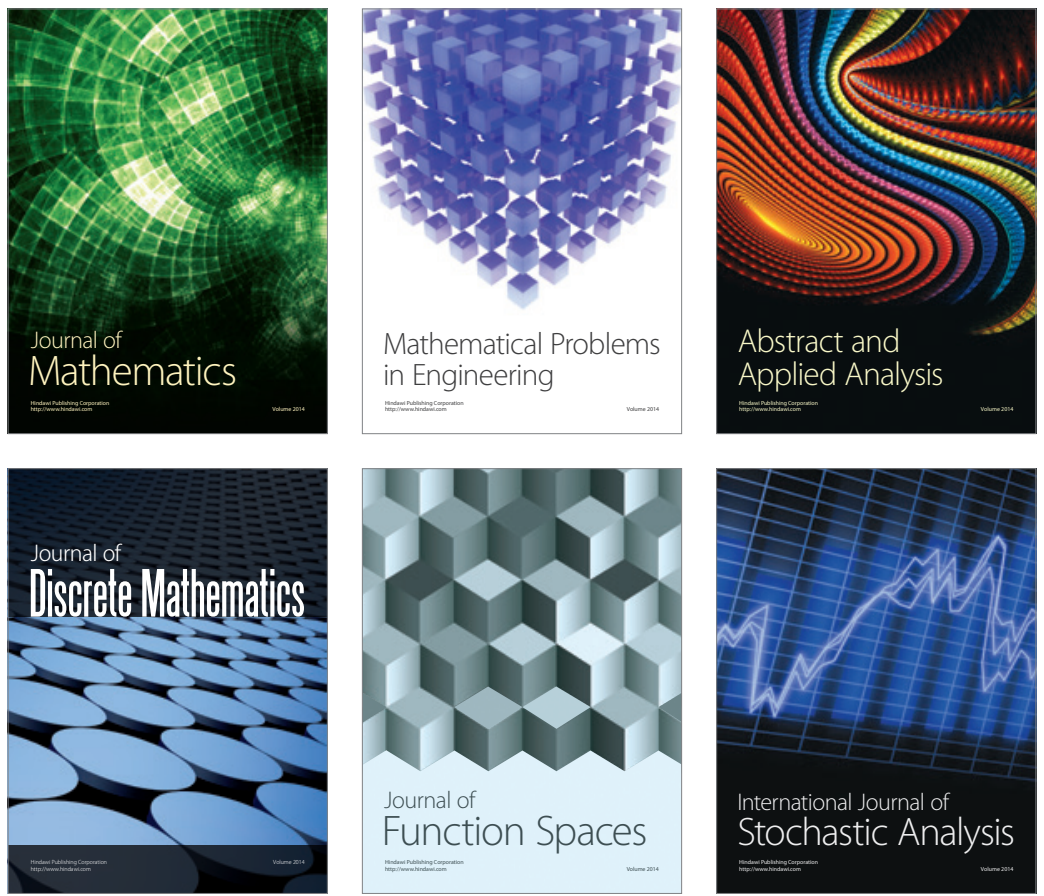

Journal of

Function Spaces

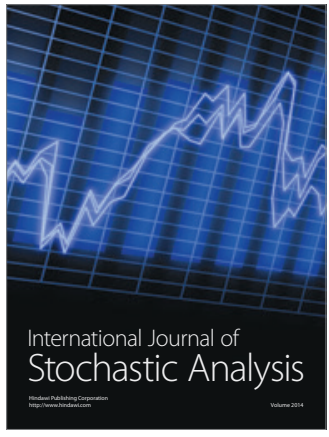

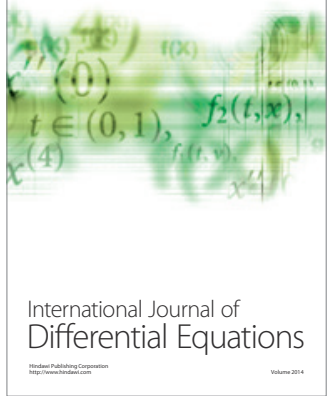
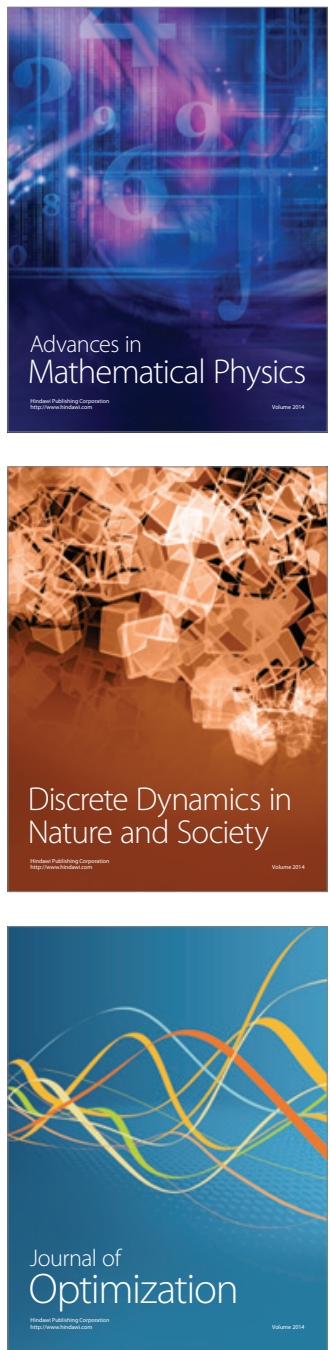